\title{
The star formation history of the Fornax dwarf spheroidal galaxy
}

\author{
Enrico V. Held ${ }^{1}$, Eline Tolstoy ${ }^{2}$, Luca Rizzi ${ }^{3}$, Mary Cesetti ${ }^{1}$, \\ Andrew A. Cole ${ }^{4}$, Giuseppina Battaglia ${ }^{5}$, Gary S. Da Costa ${ }^{6}$, \\ Marco Gullieuszik ${ }^{1}$, Mario Mateo ${ }^{7}$, Edward W. Olszewski ${ }^{8}$ \\ and Matthew G. Walker ${ }^{9}$ \\ ${ }^{1}$ Osservatorio Astronomico di Padova, INAF email: enrico.held@oapd.inaf .it \\ ${ }^{2}$ Kapteyn Astronomical Institute, Groningen \\ ${ }^{3}$ Joint Astronomy Centre, Hilo, USA \\ ${ }^{4}$ School of Mathematics and Physics, University of Tasmania, Hobart, Australia \\ ${ }^{5}$ European Southern Observatory, Garching, Germany \\ ${ }^{6}$ Research School of Astronomy and Astrophysics, Australian National University \\ ${ }^{7}$ Department of Astronomy, University of Michigan, Ann Arbor, MI 48109-1042, USA \\ ${ }^{8}$ Steward Observatory, The University of Arizona, Tucson, AZ, USA \\ ${ }^{9}$ Institute of Astronomy, University of Cambridge, United Kingdom
}

\begin{abstract}
We present the first results of a comprehensive HST study of the star-formation history of Fornax dSph, based on WFPC2 imaging of 7 Fornax fields. Our observations reach the oldest main-sequence turnoffs, allowing us to address fundamental questions of dwarf galaxy evolution, such as the spatial variations in the stellar content, and whether the old stellar population is made up of stars formed in a very early burst or the result of a more continuous star formation.
\end{abstract}

\section{Observations and reduction}

Observations of the Fornax dSph galaxy were obtained with the WFPC2 on-board the Hubble Space Telescope between Oct. 2007 and Aug. 2008. Fornax dSph is known to host a complex mix of stellar populations which varies across the galaxy (e.g., Battaglia et al. 2006). In order to obtain a spatially resolved view of the star formation in Fornax, we observed 7 regions of the galaxy designed to sample stellar populations dominated by stars formed in different epochs. The instrumental signatures were removed with on-thefly reduction by the STSci Archive pipeline. The pre-reduced images were analysed using HSTPHOT (Dolphin 2009) with the most recent calibration and corrections for the known charge transfer efficiency (CTE) problem. The calibration presented here is preliminary, and a detailed comparison of the internal precision and zero-point calibration of our HST catalogues will be presented elsewhere.

\section{The stellar populations of Fornax}

The first results of our HST study of the stellar populations of Fornax dSph are shown in Fig. 1. The overall star formation history in Fornax is illustrated by the colourmagnitude diagram (CMD) of all fields together. Our HST photometry reaches well below the main-sequence turnoff of the oldest stars, coeval to Galactic globular clusters. The subgiant branch is spread over a large interval of time, indicating an extended history 

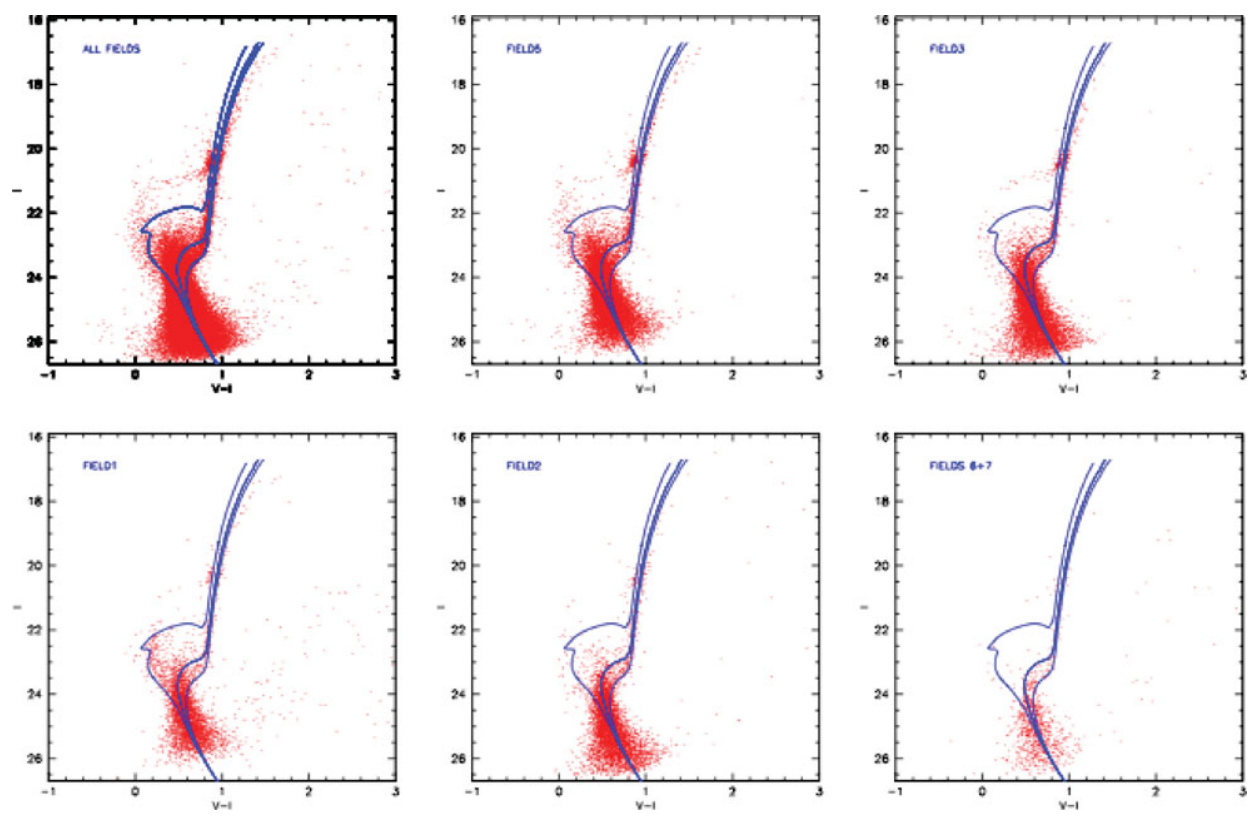

Figure 1. Colour-magnitude diagrams of the regions of Fornax observed with WFPC2. The first, upper-left panel is the sum CMD including all WFPC2 fields. Field 5 is close to the centre; Field 3 is located at an intermediate distance from the Fornax centre ( 18 arcmin). Field 1 corresponds to the inner clump detected by Coleman et al. (2004), while Field 2 is an adjacent region observed to provide a comparison field; Fields $6+7$ shows the sum CMD of two fields located quite far from the centre $(33-38$ arcmin $)$. We have superimposed theoretical isochrones from Marigo et al. (2008) with metallicity $Z=0.001$ and age 2, 7, and 12.6 Gyr.

of star formation. Other sharp features are the red clump and horizontal branch, shown with unprecedented detail, and an extended main sequence of young stars up to $\sim 1$ Gyr ago. The spatial variation of the stellar populations in Fornax is evident in Fig. 1. The CMD of the outermost fields shows only stars with age between 7-12 Gyr. Most stars in other Fornax fields belong to an extended period of star formation between 3 and 12 Gyr ago. This old and intermediate-age population is seen in the inner and intermediate fields, while star younger than 2 Gyr are found only in the innermost region. The clump detected by Coleman et al. (2004) differs from an adjacent comparison field for the presence of stars younger than $2 \mathrm{Gyr}$, in agreement with Olszewski et al. (2006). A full description of the star formation history in the different regions of Fornax will be given in a forthcoming paper (E. V. Held et al. 2009, in prep.).

\section{References}

Battaglia, G., et al. 2006, A\& A, 459, 423

Coleman, M., Da Costa, G. S., Bland-Hawthorn, J., Martínez-Delgado, D., Freeman, K. C., \& Malin, D. 2004, AJ, 127, 832

Dolphin, A. E. 2009, PASP, 121, 655

Marigo, P., Girardi, L., Bressan, A., Groenewegen, M. A. T., Silva, L., \& Granato, G. L. 2008, $A \mathscr{G} A, 482,883$

Olszewski, E. W., Mateo, M., Harris, J., Walker, M. G., Coleman, M. G., \& Da Costa, G. S. 2006, AJ, 131, 912 